Mens

revue d'histoire intellectuelle de l'Amérique française

Gérard Bouchard. Raison et contradiction. Le mythe au

secours de la pensée. Éditions Nota bene / CEFAN, Québec, 2003.

$131 \mathrm{p}$.

\title{
Frédéric Boily
}

Volume 6, numéro 1, automne 2005

URI : https://id.erudit.org/iderudit/1024263ar

DOI : https://doi.org/10.7202/1024263ar

Aller au sommaire du numéro

Éditeur(s)

Centre de recherche en civilisation canadienne-française

ISSN

1492-8647 (imprimé)

1927-9299 (numérique)

Découvrir la revue

Citer ce compte rendu

Boily, F. (2005). Compte rendu de [Gérard Bouchard. Raison et contradiction. Le mythe au secours de la pensée. Éditions Nota bene / CEFAN, Québec, 2003.

131 p.] Mens, 6(1), 126-130. https://doi.org/10.7202/1024263ar 
nous-mêmes » (p. 100). Néanmoins, Tardivel était lui-même né aux États-Unis de parents européens. Dans son ouvrage très détaillé, Rudin évite d'aborder la difficile question de savoir si les habitants de la province voyaient leur identité comme étant statique ou en mouvement. Toutefois, il fournit une description fascinante de la manière dont quelques-uns des bâtisseurs d'identité les plus assidus ont agi dans le Québec du tournant du siècle.

\section{E. A. Heaman Département d'bistoire \\ Université McGill}

Traduction : Michel Ducharme

NDLR : La traduction francaise de cet ouvrage est parue aux Presses de l'Université Laval en 2005 sous le titre L'histoire dans les rues de Québec. La célébration de Champlain et de $\mathrm{M}^{\mathrm{gr}}$ de Laval 1878-1908.

\section{Gérard Bouchard. Raison et contradiction. Le mythe au secours de la pensée. Éditions Nota bene / CEFAN, Québec, 2003. 131 p.}

Ce court ouvrage, qui est la reproduction d'une conférence donnée à l'hiver 2003 à l'Université Laval, paraittra familier à ceux ayant lu l'étude que Gérard Bouchard a consacrée à Lionel Groulx. En effet, l'historien saguenayen reprend ici, tout en les approfondissant, certains des éléments théoriques qui lui ont permis d'examiner la pensée du chanoine. 
L'analyse repose essentiellement sur l'idée que le mythe et la notion d'imaginaire collectif ne sont pas des concepts négatifs ou péjoratifs (p. 23), c'est-à-dire qu'ils ne sont pas symptomatiques d'une "dégradation de pensée » ou d'un discours fallacieux. Aux yeux de Bouchard, le mythe est un « rouage médiateur » entre les pratiques discursives (la science et les arts par exemple) et " tout ce qui loge en amont du discours " (pp. 24-25). Le mythe aurait donc une fonction médiatrice permettant à la pensée de prendre forme, si l'on peut dire, et de se réconcilier avec le réel, ce qu'elle ne peut faire avec les seules forces de la raison.

En ce sens, Bouchard écrit qu'il «aborde le mythe non pas comme produit de l'inconscient ou de la raison folle, mais en référence avec l'exercice ordinaire de la raison dans la construction de la pensée » (p. 29). Certes, explique-t-il, le mythe a pour «caractéristique principale $[. .$.$] de n'être pas vérifia-$ ble », principalement parce qu'il est motivé par des émotions, des croyances et des intérêts (p. 28). Néanmoins, ce qui importe c'est que, comme producteur de sens, le mythe est un «levier» permettant à la raison de surmonter les contradictions et de les dépasser (p. 42). C'est pourquoi on ne doit pas juger le mythe au seul étalon de la vérité mais à celui de l'efficacité. Par là, il faut comprendre qu'un mythe efficace permet à la pensée de surmonter les inévitables contradictions qui surgissent lorsqu'elle subit l'épreuve du réel. On se retrouverait, avance Bouchard, devant « trois scénarios possibles » quant à la façon dont les systèmes rationnels s'y prennent pour surmonter les «éléments contradictoires » qui sont présents originellement dans les discours.

Il y aurait d'abord ce que Bouchard appelle la "pensée radicale ", laquelle parvient à surmonter les contradictions de manière efficace parce que celles-ci sont tout simplement éradiquées. Telle est la solution avancée par les révolution- 
naires français, comme Saint-Just qui préconise «la destruction totale de ce qui est opposé [à la République]» (p. 48). Le compromis est absent de cette forme de pensée, la Raison s'érigeant ici en grande ordonnatrice du monde social. Si le totalitarisme constitue la forme extrême de la pensée radicale, Bouchard croit aussi que l'idéologie patriote (et certaines formes de la pensée souverainiste) en fait partie. On aurait aimé en savoir un peu plus ici : après tout, on peut s'étonner de voir les Patriotes côtoyer le totalitarisme dans une même catégorie analytique. $\mathrm{Il}$ y a certes une volonté révolutionnaire dans les insurrections, mais les Patriotes étaient-ils si radicaux? Des éclaircissements auraient été souhaitables.

La "pensée organique ", quant à elle, parvient à surmonter les contradictions mais en évitant les bains de sang trop souvent caractéristiques des entreprises radicales ou utopiques. L'univers intellectuel de la pensée organique, ouvert à la complexité, ne cherche pas à éradiquer la contradiction mais à la surmonter au moyen de mythes médiateurs, c'est-àdire des mythes qui, par une espèce de ruse de la raison (et du mythe), laissent croire que les contradictions ont été ou peuvent être surmontées. Aux yeux de Bouchard, le meilleur exemple que l'on puisse donner d'une telle attitude est celui de l'American dream, où chacun est maître de son destin et libre en conséquence de construire sa propre destinée. Or, ce mythe a fonctionné dans une société où les clivages de toutes sortes ont été importants, qu'on pense seulement à la ségrégation raciale. Le Canada anglais loge aussi à l'enseigne de la pensée organique puisqu'on y est parvenu à réconcilier l'irréconciliable à savoir l'idée d'indépendance nationale et celle du maintien du lien colonial. La pensée organique est donc familière (et c'est la clé de son succès) des "assemblages" apparemment contradictoires, mais porteurs d'un projet mobilisateur. 
Ce n'est pas le cas de la pensée équivoque, troisième forme identifiée par Bouchard, qui ne parvient pas à proposer de synthèse mobilisatrice, car elle reste en quelque sorte figée devant la complexité. Incapable « de harnacher [1]es contradictions » (p. 81), la pensée équivoque se contente d'une juxtaposition d'affirmations contraires sans qu'aucune solution satisfaisante pour l'action ne soit proposée. Elle est tout simplement signe d'impuissance comme le montre l'exemple de Lionel Groulx qui ne s'est jamais décidé quant à l'avenir politique du Québec. Alors que la pensée organique propose des «mythes projecteurs" - des mythes porteurs de changement - la pensée équivoque se contente de "mythes dépresseurs ", lesquels inhibent l'action et conduisent presque inexorablement à l'échec tant ils sont éloignés de la réalité. En somme, selon Bouchard, la pensée organique est de loin supérieure sur le plan de l'efficacité, tout particulièrement sur le "long terme », aux pensées équivoque et radicale (p. 88).

Ambitieux, l'historien conclut en invitant les chercheurs à une relecture de la modernité, c'est-à-dire à revenir au moment où l'on assiste à la « déchirure irréparable entre la raison et le mythe " (p. 116). Ce retour aux origines devrait permettre d'établir la pertinence du mythe pour aujourd'hui et, plus précisément, la nécessité de " réconcilier mythe et raison » ( $\mathrm{p}$. 117). En d'autres termes, à une "nouvelle alliance " laquelle implique de "réenchanter» le monde au moyen du mythe, encore que Bouchard semble croire que le mythe n'a peutêtre jamais vraiment disparu de notre horizon intellectuel.

Mais le monde est-il si désenchanté qu'il faille absolument le réenchanter? Certes, le religieux n'est plus au fondement de l'existence sociale et en cela il y a bien désenchantement du monde ou sortie de la religion pour le dire à la manière de Marcel Gauchet. Toutefois, certains sociologues des 
religions, comme Danièle Hervieu-Léger, font remarquer que les transformations du croire ne signifient pas la disparition du croire. En ce sens, le monde demeure peut-être encore plus enchanté qu'il n'y paraît de prime abord ("L'Église est morte, vive la religion!» Le Monde des débats, décembre 2000, pp. 16-17). Admettons cependant avec Bouchard que, sur le plan de la représentation globale, notre monde soit sur l'inéluctable pente du désenchantement. Doit-on pour autant conclure qu'il faille réinventer le politique à partir du mythe, comme semble nous y inviter Bouchard (même si ce n'est pas dit textuellement)? Le $\mathrm{XX}^{\mathrm{e}}$ siècle, si on considère les ravages occasionnés par les religions politiques (le fascisme pour n'en nommer qu'une), ne montre-il pas tous les dangers d'une telle attitude? Compte tenu du triste bilan du siècle qui vient de s'achever, d'aucuns, inspirés par les analyses d'Ernst Cassirer et de son ouvrage Le Mythe de l'État, pourraient conclure qu'il faut penser et surtout réinventer la politique contre les puissances d'enchantement, c'est-à-dire contre le mythe plutôt qu'avec lui. C'est à ce genre d'objection que devra maintenant répondre Bouchard, ce qui devrait le conduire à expliquer avec plus d'autorité pourquoi il plaide pour une nouvelle alliance du mythe et de la raison. Voilà qui annonce de passionnantes discussions.

Frédéric Boily

Faculté Saint-Jean University of Alberta 